\title{
Fecal calprotectin levels in Helicobacter pylori gastritis in children
}

\author{
Özlem Yüksel Aksoy ${ }^{1 \oplus}$, Oğuz Canan²ْ, Ferda Özbay Hoşnut ${ }^{3 \oplus}$, \\ EdaYılmaz Akçay ${ }^{4 \oplus}$, Figen Özçay ${ }^{5 \odot}$
}

${ }^{1}$ Department of Pediatric Nephrology, Kayseri City Hospital, Kayseri; ${ }^{2}$ Department of Pediatric Gastroenterology, Başkent University Faculty of Medicine, Adana; ${ }^{3}$ Department of Pediatric Gastroenterology, Dr. Sami Ulus Maternity, Children's Health and Diseases Training and Research Hospital, Ankara; Departments of ${ }^{4}$ Pathology and ${ }^{5}$ Pediatric Gastroenterology, Başkent University Faculty of Medicine, Ankara, Turkey.

\begin{abstract}
Background. Fecal calprotectin is an important inflammatory marker in intestinal diseases and is not routinely used in the upper gastrointestinal system disorders. The aim of this study was to show whether there is a relationship between fecal calprotectin levels and Helicobacter pylori ( $H$ pylori) gastritis in children and to determine the association of fecal calprotectin levels with gastric biopsy results in terms of chronic inflammation and neutrophil activity.

Methods. Patients with the complaints of the upper gastrointestinal system (epigastric pain, heartburn, nausea and vomiting) who were planned to undergo endoscopy were enrolled prospectively. The presence of $H$ pylori was defined according to the gastric antrum biopsy results. Fecal calprotectin level was tested in the stool sample of the patients. The fecal calprotectin levels, upper gastrointestinal endoscopy and gastric biopsy results of 89 patients were evaluated.
\end{abstract}

Results. $H$ pylori was found to be positive in the gastric biopsies of $51(57.3 \%)$ patients. In the $H$ pylori positive group mean fecal calprotectin level was $74.8 \pm 67 \mu \mathrm{g} / \mathrm{g}$, and in the $\mathrm{H}$ pylori negative group mean fecal calprotectin level was $52.7 \pm 46 \mu \mathrm{g} / \mathrm{g}$ and the difference was significant ( $\mathrm{p}=0.039$ ). We also found a significant relationship between fecal calprotectin levels and gastric neutrophil activity grades $(\mathrm{p}=0.034)$.

Conclusions. Mean fecal calprotectin levels were found to be higher in $H$ pylori positive subjects in our study. Fecal calprotectin levels were correlated with gastric neutrophil activity grades. Fecal calprotectin represents gastric neutrophilic inflammation. When interpreting a high fecal calprotectin level, $H$ pylori infection should be kept in mind.

Key words: fecal calprotectin, gastritis, gastric biopsy, Helicobacter pylori, child, pediatric.

Helicobacter pylori (H pylori) is the major cause of chronic gastritis and peptic ulcer disease, and classified as a group 1 carcinogen by the World Health Organisation. ${ }^{1}$ H pylori infection is usually acquired during childhood via fecal-oral or oral-oral routes. ${ }^{2}$ Most of the infected children are asymptomatic. Pediatric prevalence of the infection is $10 \%$ in developed countries, whereas it is more than $50 \%$ in developing countries and the prevalence

$\triangle$ Özlem Yüksel Aksoy

ozlem_yurtsever@yahoo.com

Received 5th March 2020, accepted 4th April 2020. increases with age. ${ }^{3,4}$ In a group of children who had upper gastrointestinal system symptoms and underwent endoscopy, the incidence of $H$ pylori was reported as $51.8 \% .^{5}$ The most accurate diagnostic method for detecting $H$ pylori is showing the microorganism in the gastric endoscopic biopsies. ${ }^{6}$

$H$ pylori infection usually presents as antral gastritis or gastric and duodenal ulcers in children. Chronic gastritis is found in almost every child infected with $H$ pylori; whereas peptic ulcer develops in only $10 \% .{ }^{7,8}$ Alterations in the infected gastric mucosa may result in 
atrophy, metaplasia and cancer in years. ${ }^{9}$

There is an evolution of the inflammatory response to $H$ pylori as the infection persists over time. In the early stages of $H$ pylori infection, polymorphonuclear cell infiltration in the gastric mucosa is the major histopathological finding in adults, whereas lymphocytes predominate with the progression of the disease. ${ }^{10}$ In children, although the lymphocytic response to $H$ pylori appears to be the predominant component, active chronic gastric inflammation with neutrophils is also detected. ${ }^{10,11}$

In gastric biopsies of almost all cases with $H$ pylori, neutrophils are detected; neutrophil activity is a common feature of $H$ pylori gastritis. ${ }^{12}$ Neutrophils are usually located in the lamina propria, within the epithelium and the foveolar lumen. The extent of mucosal injury and the intensity of $H$ pylori infection is associated with the density of intraepithelial neutrophils. ${ }^{12-14}$

Calprotectin, a calcium and zinc binding protein which is mainly released from the neutrophils, increases in many inflammatory processes as a sign of the neutrophil chemotaxis. ${ }^{15}$ Fecal calprotectin is an objective, non-invasive marker of the intestinal inflammation, commonly used in the diagnosis and follow-up of inflammatory bowel diseases. ${ }^{16}$ However there are very few studies that investigate the relationship between fecal calprotectin levels and the upper gastrointestinal system diseases in adults and children. ${ }^{17,18}$

The aim of this study was to show whether fecal calprotectin levels increase in $H$ pylori infection, and to determine the relationship between gastric neutrophil activity and fecal calprotectin levels in pediatric patients.

\section{Material and Methods}

\section{Study Population}

Children with complaints of the upper gastrointestinal system, such as epigastric pain, pyrosis, heart burn, nausea, vomiting, and halitosis, who applied to the Pediatric Gastroenterology Departments of Baskent University, Faculty of Medicine and Dr. Sami Ulus Maternity, Children's Health and Diseases, Training and Research Hospital were evaluated between November 2013 and May 2014.

Children who were older than five years of age, who had not used proton pump inhibitors or antibiotics within the last 15 days, and who were planned to undergo upper gastrointestinal endoscopic examination were enrolled as the study group. Children with chronic liver, kidney, rheumatological diseases and celiac disease patients were excluded.

We also excluded patients who had endoscopic mucosal lesions such as esophagitis, gastric ulcer, gastric polyps, duodenal ulcers in order to prevent their possible effects on fecal calprotectin levels. Fecal calprotectin test was performed in 89 patients who fulfill all these criteria mentioned above.

Direct microscopic examination of the stool to exclude parasites, and fecal calprotectin measurement were performed within two days of the endoscopy.

Only patients older than five years of age were enrolled in the study due to the requirement of being able to define the symptoms and answer the questions related to their gastrointestinal complaints. Besides, fecal calprotectin values are known to be higher in infants, and the cut-off value of fecal calprotectin showing inflammation has not been studied well enough in patients younger than 5 years of age. ${ }^{19}$ Previous studies have demonstrated that the fecal calprotectin levels do not differentiate between the age groups after the age of four, and $50 \mu \mathrm{g} / \mathrm{g}$ level may be used as a cut-off value for mucosal inflammation. ${ }^{20}$

Histopathological examination of the gastric mucosa was used as a diagnostic method to show the presence of $H$ pylori. Gastric antral biopsies were evaluated according to The Sydney Histopathologic Classification ${ }^{12}$, 
by two pathologists who were blinded to the fecal calprotectin results of the patients. Hematoxylen-Eosin and Giemsa dyes were used for the examination of the specimens. According to the endoscopic gastric antral biopsy results, the subjects were divided into two groups, $H$ pylori positive and negative groups. Fecal calprotectin levels were compared between $H$ pylori positive and negative groups. Chronic inflammation, neutrophil activity and $H$ pylori intensity grades were also compared with fecal calprotectin levels.

This study was conducted with the approval of the Ethical Committee of Baskent University Faculty of Medicine on 02/10/2013 (Project number: KA 13/196). Informed consent were taken from families. Baskent University Research Foundation supported the study.

\section{Fecal Calprotectin Measurement}

Stool specimens were taken within two days of the endoscopic procedure. Quantum Blue Calprotectin ${ }^{\circledR}$ BÜHLMANN rapid test was used for fecal calprotectin measurement. Quantitative results are available with this rapid test, and the results are comparable with ELISA test. Fecal calprotectin level is measured between $30-300 \mathrm{mcg} / \mathrm{g}$ and the cut-off value is $50 \mathrm{mcg} / \mathrm{g}$. With this cut-off value the sensitivity and the specificity of the test for differentiating organic and functional disorders, are $84.4 \%$ and 94.5 respectively.

The quantitative rapid test uses lateral flow technology, calprotectin reader analyzes the signal intensity from the test and control line to give a quantitative value. ${ }^{21}$ Stool extract is diluted with buffering solution, and centrifugated; supernatant part is used for analysis.

\section{Statistical Analysis}

Preliminary statistical analysis showed that the number of the subjects needed to compare the $H$ pylori positive and negative groups, were a minimum of 38 patients for each group.
The results of 38 patients in $H$ pylori negative group, and 51 patients in $H$ pylori positive group were evaluated with SPSS for Windows 11.5.

For the analysis of the categorical data of $H$ pylori positive and negative groups, $\chi^{2}$ test and Fisher-Exact tests were used. Student $t$ test was performed for normally distributed data, and Mann-Whitney $U$ test for non-normally distributed data. Spearman Rank Correlation analysis and Kruskal-Wallis tests were used for the relations with fecal calprotectin.

Frequencies and percentages were used as descriptive values in the categorical data. Arithmetical mean \pm standard deviation was used for the normally distributed data, and median and interquartile range (IQR) were used for the non-normally distributed data.

Statistical significance was accepted as 0.05.

\section{Results}

The fecal calprotectin levels of 89 patients (70 (78.7\%) girls, and 19 (21.3\%) boys) who underwent upper gastrointestinal endoscopy were evaluated. $H$ pylori infection was found in the gastric biopsies of 51 (57.3\%) patients, whereas in $38(42.7 \%)$ patients $H$ pylori was negative. The mean age was $12.8 \pm 3$ years in the $H$ pylori positive group, and $13.11 \pm 2.4$ years in the $H$ pylori negative group ( $\mathrm{p}>0.05)$.

The main presenting symptoms were epigastric pain in $55(61.8 \%)$ patients, abdominal pain in $13(14.6 \%)$ patients, heartburn (pyrosis) in $12(13.5 \%)$ patients, and nausea in seven (7.9\%) patients. Halitosis in one, and epigastric fullness in another patient were recorded as chief symptoms (Table I).

When we performed detailed symptom inquiry, $71(79.8 \%)$ patients denoted epigastric pain, and $46(52 \%)$ patients expressed pyrosis. There was not a difference in epigastric pain and pyrosis frequency in terms of $H$ pylori status ( $p>0.05$ ). Nausea and/or vomiting were stated in 29 $(34.5 \%)$ patients and this symptom was more 
Table I. Main symptoms of the patients at presentation.

\begin{tabular}{|c|c|c|c|}
\hline & \multicolumn{2}{|c|}{ Hp presence } & \multirow{2}{*}{ Total } \\
\hline & $\mathrm{Hp}(-)$ & $\mathrm{Hp}(+)$ & \\
\hline Epigastric pain (n) & 23 & 32 & 55 \\
\hline$\%$ & $60.5 \%$ & $62.7 \%$ & $61.8 \%$ \\
\hline Heartburn(pyrosis) (n) & 4 & 8 & 12 \\
\hline$\%$ & $0.5 \%$ & $15.7 \%$ & $13.5 \%$ \\
\hline Stomachache (n) & 5 & 8 & 13 \\
\hline$\%$ & $13.2 \%$ & $15.7 \%$ & $14.6 \%$ \\
\hline Nausea (n) & 5 & 2 & 7 \\
\hline$\%$ & $13.2 \%$ & $\% 3.9$ & $\% 7.9$ \\
\hline Halitosis (n) & 0 & 1 & 1 \\
\hline$\%$ & $0 \%$ & $2.0 \%$ & $1.1 \%$ \\
\hline Fullness (n) & 1 & 0 & 1 \\
\hline$\%$ & $2.6 \%$ & $0 \%$ & $1.1 \%$ \\
\hline Total & 38 & 51 & 89 \\
\hline
\end{tabular}

frequent (20 vs. 9) in H pylori positive patients $(\mathrm{p}<0.05)$.

When the endoscopic diagnoses were evaluated, $36(40 \%)$ subjects were found to have normal upper endoscopic findings, 24 (27\%) had antral nodular gastritis, 21 (24\%) had erythematous gastritis, $6(7 \%)$ had antral superficial gastritis, $2(2 \%)$ had erosive gastritis.

In $51 \mathrm{H}$ pylori positive patients, 21 (41\%) had antral nodular gastritis. Twenty one out of 24 $(87.5 \%)$ patients with antral nodular gastritis were found to be positive for $H$ pylori. The relationship between antral nodular gastritis and $H$ pylori was statistically significant ( $\mathrm{p}$ $<0.001$ ) (Table II).

Mean fecal calprotectin level of all patients was $65.9 \pm 60.3 \mathrm{mcg} / \mathrm{g}$ (min: 10 , max: $300 \mathrm{mcg} / \mathrm{g}$ ). Mean fecal calprotectin levels of $H$ pylori positive and negative groups were $74.8 \pm 67 \mu \mathrm{g} / \mathrm{g}$, and 52.7 $\pm 46 \mu \mathrm{g} / \mathrm{g}$, respectively. The difference between the two groups was statistically significant $(\mathrm{p}=$ 0.039) (Table III).

The cut-off value for fecal calprotectin for this study was calculated as $30.49 \mu \mathrm{g} / \mathrm{g}$ with ROC (receiver operating character) analysis. According to this cut-off value, for the detection

Table II. Endoscopic diagnoses and H pylori.

\begin{tabular}{|c|c|c|c|}
\hline & Hp (-) & $\mathrm{Hp}(+)$ & Total \\
\hline Normal (n) & 18 & 18 & 36 \\
\hline$\%$ & $47.7 \%$ & $35.3 \%$ & $40.4 \%$ \\
\hline Antral nodular gastritis (n) & 3 & 21 & 24 \\
\hline$\%$ & $7.9 \%$ & $41.2 \%$ & $27 \%$ \\
\hline Antral superficial gastritis (n) & 6 & 0 & 6 \\
\hline$\%$ & $15.8 \%$ & $0 \%$ & $6.7 \%$ \\
\hline Erosive gastritis (n) & 2 & 0 & 2 \\
\hline$\%$ & $5.3 \%$ & $0 \%$ & $2.2 \%$ \\
\hline Erythematous gastritis (n) & 9 & 12 & 21 \\
\hline$\%$ & $23.7 \%$ & $23.5 \%$ & $23.6 \%$ \\
\hline Total & 38 & 51 & 89 \\
\hline
\end{tabular}


Table III. The relationship between fecal calprotectin and Hp infection ( $\left.{ }^{*} \mathrm{p}=0.039\right)$.

\begin{tabular}{lcc}
\hline Fecal calprotectin $(\mu \mathrm{g} / \mathrm{g})$ & $\mathrm{Hp}(-) \mathrm{n}=38$ & $\mathrm{Hp}(+) \mathrm{n}=51$ \\
\hline Mean* & 52.7 & 74.8 \\
Standard deviation & 46.8 & 67.6 \\
Median & 29.9 & 40 \\
Minimum & 29.9 & 29.9 \\
Maximum & 222 & 300 \\
Interquartile Range $(\mathrm{IQR})$ & 27.6 & 86 \\
\hline
\end{tabular}

of $\mathrm{H}$ pylori, the sensitivity and the specificity of fecal calprotectin testing were $60.8 \%$ and $63.2 \%$ respectively.

The possibility of fecal calprotectin level being more than $30.49 \mu \mathrm{g} / \mathrm{g}$ in H pylori positive patients was 2.657 times higher than $H$ pylori negative patients (Odds ratio 2.657, CI: 95\%, 1.117-6.319).

However, the recommended cut-off value to determine the presence of intestinal inflammation is accepted as $50 \mu \mathrm{g} / \mathrm{g}$ in Quantum Blue Calprotectin ${ }^{\circledR}$ BÜHLMANN rapid test. When we use this cut-off level, the sensitivity and the specificity of fecal calprotectin to detect H pylori were $39.2 \%$ and $73.7 \%$ respectively.

We evaluated gastric biopsy results in terms of chronic inflammation, neutrophil activity, $H$ pylori intensity, intestinal metaplasia and atrophy according to the updated Syndey Classification. ${ }^{12}$

Out of $38 \mathrm{H}$ pylori negative children, 7 (18\%) cases had normal findings in their gastric biopsies. Mean fecal calprotectin level in those with normal gastric biopsies was $53.8 \pm 31.04$ $\mu \mathrm{g} / \mathrm{g}$. Remaining 31(82\%) patients reported as having gastritis in their gastric biopsies, despite not having $H$ pylori. Mean fecal calprotectin in that group was $52.4( \pm 50.09) \mu \mathrm{g} / \mathrm{g}$. The difference in mean fecal calprotectin levels between those two groups was not significant.

All $51 \mathrm{H}$ pylori positive patients had some degree of chronic inflammation as mild in 18, moderate in 24 or severe in 9 cases. Chronic inflammation of the gastric antral mucosa was seen in $30 / 38$ (79\%) of $H$ pylori negative patients as mild in 26 , moderate in 4 cases. Severity of chronic inflammation was higher in $H$ pylori positive group $(\mathrm{p}<0.001)$ than the $H$ pylori negative group.

Gastric neutrophil activity was seen in 40/51 (78\%) $H$ pylori positive patients (mild 17, moderate 19, and severe in 4 cases). Neutrophil activity was seen only in 12 out of 38 (32\%) H pylori negative patients. When we compared two groups, gastric neutrophil activity grades were higher in H pylori positive group $(\mathrm{p}<0.001)$.

There was no relationship between fecal calprotectin levels and chronic inflammation grades $(p=0.093)$, however, we found a significant relationship between fecal calprotectin levels and gastric neutrophil activity grades $(p=0.034)$ (Table IV).

$H$ pylori intensity was mild in $22(43 \%)$, moderate in $17(33 \%)$, and severe in $12(\% 24)$ patients with $H$ pylori infection. When the relationship between fecal calprotectin levels and $H$ pylori intensity in gastric biopsy samples were evaluated; although $H$ pylori intensity grade and fecal calprotectin median levels are proportional, this relationship was not statistically significant $(p=0.197)$ (Table IV).

Intestinal metaplasia was not seen in any of the subjects. Gastric atrophy was seen in 12 (13\%) children (3 H pylori negative and $9 \mathrm{H}$ pylori positive).

\section{Discussion}

$H$ pylori is one of the world's most common infectious diseases and half of the world's population is infected with it. $H$ pylori is usually 
Table IV. The relationship between fecal calprotectin levels and the grades of chronic inflammation, neutrophil activity, and Hp intensity.

\begin{tabular}{|c|c|c|c|c|c|}
\hline & 0 (none) & 1(mild) & 2 (moderate) & 3 (severe) & P value \\
\hline $\begin{array}{l}\text { A. Chronic inflammation } \\
\text { Fecal calprotectin }(\mu \mathrm{g} / \mathrm{g})\end{array}$ & & & & & 0.093 \\
\hline Mean & 74 & 48 & 81 & 89 & \\
\hline Median & 50.4 & 29 & 44 & 29.9 & \\
\hline Minimum & 29 & 29 & 10 & 29 & \\
\hline Maximum & 222 & 220 & 300 & 243 & \\
\hline $\begin{array}{l}\text { B.Neutrophil activity } \\
\text { Fecal calprotectin }(\mu \mathrm{g} / \mathrm{g})\end{array}$ & & & & & 0.034 \\
\hline Mean & 54 & 58 & 74.8 & 164 & \\
\hline Median & 30 & 29.9 & 43 & 178.5 & \\
\hline Minimum & 29 & 29 & 10 & 58 & \\
\hline Maximum & 300 & 222 & 243 & 242 & \\
\hline $\begin{array}{l}\text { C. Hp intensity } \\
\text { Fecal calprotectin }(\mu \mathrm{g} / \mathrm{g})\end{array}$ & & & & & 0.197 \\
\hline Mean & 52.7 & 60 & 86 & 86 & \\
\hline Median & 29.9 & 32 & 43 & 44 & \\
\hline Minimum & 29.9 & 29.9 & 30 & 29.9 & \\
\hline Maximum & 222 & 141 & 300 & 242 & \\
\hline
\end{tabular}

acquired during childhood, infection rates remain higher in some groups of children, dependent on factors such as low socioeconomic status and bad sanitary conditions. ${ }^{22}$

Epigastric pain, nausea/vomiting, regurgitation, pyrosis, abdominal pain are the symptoms of $H$ pylori infection in children, whereas the infection may also be asymptomatic. In our study group consisting of 89 patients with upper gastrointestinal symptoms, 51 (57\%) of the subjects were found to have $H$ pylori infection. Although we excluded the duodenal and gastric ulcer patients, $H$ pylori prevalence is still high when compared with the literature. ${ }^{18,23}$

In a study in China, 1634 children underwent upper endoscopic evaluation due to gastrointestinal system symptoms, and $H$ pylori prevalence was found to be $32.1 \%$. Active inflammation in gastric biopsies was shown in $26.9 \%$ of $H$ pylori positive patients, whereas it was only $4.1 \%$ in $H$ pylori negative patients. ${ }^{24}$ In our study, we found higher rates of active gastric inflammation apart from $H$ pylori status, and significant difference in terms of gastric neutrophil activity between $H$ pylori positives $(78 \%)$ and negatives (32\%). These different results could be explained by other environmental factors and ethnic differences.

Studies searching about the utilization of fecal calprotectin in the diagnosis of gastritis are very limited. Montalto et al. ${ }^{17}$ evaluated fecal calprotectin levels in histologically diagnosed 61 adult gastritis patients and 74 healthy adults. Subjects with chronic gastritis were divided into three groups having mild, moderate and severe gastritis. Fecal calprotectin levels did not differ between those three groups and healthy controls. Also, there were not any differences between the groups, related with $H$ pylori presence and chronic proton pump usage.

In a recent study in pediatric functional abdominal pain-related gastrointestinal disorders, the fecal calprotectin levels of 17 $H$ pylori positive and $39 H$ pylori negative patients were found to be similar. In this study, median fecal calprotectin levels did not also differ between cases of patients and healthy controls. ${ }^{18}$ In contrary to that study, our H pylori 
positive subjects had higher fecal calprotectin mean levels than $H$ pylori negative subjects. Nevertheless, our sample size of patients with $H$ pylori gastritis was larger and gastric neutrophilic activity was high.

Flagstadt et al. ${ }^{25}$, demonstrated low levels of fecal calprotectin in children with functional gastrointestinal disorders (FGID); fecal calprotectin levels did not differ significantly between FGID entities. However, this study was performed after excluding any organic disease such as H pylori gastritis.

In a patient group with 56 cases and quiescent Crohn disease, it was shown that fecal calprotectin levels did not change significantly with $H$ pylori eradication. ${ }^{26}$ There were only six $H$ pylori positive patients which lead them to avoid coming to a clear conclusion.

When we compared fecal calprotectin levels with chronic inflammation, neutrophil activity, and $H$ pylori intensity grades of gastric biopsies according to the updated Sydney Classification ${ }^{12}$, a significant relationship to fecal calprotectin was only shown between grades of neutrophil activity. H. pylori gastritis is characterized by the infiltration of the lamina propria between the surface epithelium, the foveolae and gland necks by plasma cells, lymphocytes, macrophages and neutrophil granulocytes. The existence and degree of neutrophils inside the epithelium or in the lamina propria determine the activity of gastritis. ${ }^{13}$ Since fecal calprotectin is a marker of neutrophilic inflammation, our result showing the significant relationship between fecal calprotectin and gastric neutrophil activity might be stated as an expected finding.

Summerton et al. ${ }^{27}$ evaluated fecal calprotectin levels in 26 patients with gastritis or duodenitis, and found normal results. Fecal calprotectin levels are expected to be higher with increasing neutrophil degree, which is a sign of activity. Contrary to previous studies we found that activity degree is related with fecal calprotectin levels. The lower fecal calprotectin levels in upper gastrointestinal diseases in previous studies might be explained by less severe inflammation and a smaller area of inflammation when compared to inflammatory bowel diseases. ${ }^{17}$

This study compares fecal calprotectin levels between $H$ pylori positive and negative patients, as well as in different degrees of inflammation, activity and $H$ pylori density in childhood gastritis. Mean fecal calprotectin levels were found to be significantly higher in $H$ pylori positive subjects in our study. We also found a relationship between neutrophil activity grades and fecal calprotectin. However, these data are not sufficient to make a statement about the certain increase of fecal calprotectin levels in childhood H pylori gastritis.

Fecal calprotectin is a non-invasive marker showing the neutrophilic inflammation. Further studies are needed to confirm our results and provide the routine usage of fecal calprotectin in patients with upper GI symptoms and in the diagnosis of H pylori.

\section{REFERENCES}

1. Schistosomes, liver flukes and Helicobacter pylori. IARC Working Group on the Evaluation of Carcinogenic Risks to Humans. Lyon, 7-14 June 1994. IARC Monogr Eval Carcinog Risks Hum 1994; 61: 1-241.

2. Roma E, Panayiotou J, Pachoula J, et al. Intrafamilial spread of Helicobacter pylori infection in Greece. J Clin Gastroenterol 2009; 43: 711-715.

3. Pérez-Pérez GI,Sack RB, Reid R, Santosham M, Croll J, Blaser MJ. Transient and persistent Helicobacter pylori colonization in Native American children. J Clin Microbiol 2003; 41: 2401-2407.

4. Ertem D, Harmanci H, Pehlivanoğlu E. Helicobacter pylori infection in Turkish preschool and school children: role of socioeconomic factors and breast feeding. Turk J Pediatr 2003; 45: 114-122.

5. Carvalho MA, Machado NC, Ortolan EV, Rodrigues MA. Upper gastrointestinal histopathological findings in children and adolescents with nonulcer dyspepsia with Helicobacter pylori infection. J Pediatr Gastroenterol Nutr 2012; 55: 523-529.

6. Guarner J, Kalach N, Elitsur Y, Koletzko S. Helicobacter pylori diagnostic tests in children: review of the literature from 1999 to 2009. Eur J Pediatr 2010; 169: 15-25. 
7. Drumm B, Koletzko S, Oderda G. Helicobacter pylori infection in children: a consensus statement. European Paediatric Task Force on Helicobacter pylori. J Pediatr Gastroenterol Nutr 2000; 30: 207213.

8. Uğraş M, Pehlivanoğlu E. Helicobacter pylori infection and peptic ulcer in eastern Turkish children: is it more common than known? Turk J Pediatr 2011; 53: 632-637.

9. Fox JG, Wang TC. Inflammation, atrophy, and gastric cancer. J Clin Invest 2007; 117: 60-69.

10. Whitney AE, Guarner J, Hutwagner L, Gold BD. Helicobacter pylori gastritis in children and adults: comparative histopathologic study. Ann Diagn Pathol 2000; 4: 279-285.

11. Bedoya A, Garay J, Sanzón F, et al. Histopathology of gastritis in Helicobacter pylori-infected children from populations at high and low gastric cancer risk. Hum Pathol 2003; 34: 206-213.

12. Dixon MF, Genta RM, Yardley JH, Correa P. Classification and grading of gastritis. The updated Sydney system. International Workshop on the Histopathology of Gastritis, Houston 1994. Am J Surg Pathol 1996; 20: 1161-1181.

13. Fiocca R, Villani L, Luinetti $\mathrm{O}$, et al. Helicobacter colinization and histopathological profile of chronic gastritis in patients with or without dyspepsia, mucosal erosion and peptic ulcer: a morphological approach to the study of ulcerogenesis in man. Virchows Arch A Pathol Anat Histopathol 1992; 420: 489-498.

14. Stolte M, Eidt S. Chronic erosions of the antral mucosa: a sequela of Helicobacter pylori-induced gastritis. Z Gastroenterol 1992; 30: 846-850.

15. Bjarnason I. The use of fecal calprotectin in inflammatory bowel disease. Gastroenterol Hepatol (N Y) 2017; 13: 53-56.

16. Burri E, Beglinger C. The use of fecal calprotectin as a biomarker in gastrointestinal disease. Expert Rev Gastroenterol Hepatol 2014; 8: 197-210.

17. Montalto M, Gallo A, Ianiro G, et al. Can chronic gastritis cause an increase in fecal calprotectin concentrations? World J Gastroenterol 2010; 16: 3406-3410.
18. Sýkora J, Huml M, Siala K, et al. Paediatric Rome III criteria-related abdominal pain is associated with Helicobacter pylori and not with calprotectin. J Pediatr Gastroenterol Nutr 2016; 63: 417-422.

19. Ezri J, Nydegger A. Pediatrics. Fecal calprotectin in children: use and interpretation. Rev Med Suisse 2011; 7: 69-70.

20. Kostakis ID, Cholidou KG, Vaiopoulos AG, Vlachos IS, Perrea D, Vaos G. Fecal calprotectin in pediatric inflammatory bowel disease: a systematic review. Dig Dis Sci 2013; 58: 309-319.

21. Lobatón T, Rodríguez-Moranta F, Lopez A, Sánchez E, Rodríguez-Alonso L, Guardiola J.A new rapid quantitative test for fecal calprotectin predicts endoscopic activity in ulcerative colitis. Inflamm Bowel Dis 2013; 19: 1034-1042.

22. Mišak Z, Hojsak I, Homan M. Review: Helicobacter pylori in pediatrics. Helicobacter 2019; 24(Suppl 1): e12639.

23. Ünlüsoy Aksu A, Yılmaz G, Eğritaş Gürkan Ö, Sarı S, Dalgıç B. The effect of Helicobacter pylori eradication on functional dyspepsia in Turkish children. Helicobacter 2018; 23: e12497.

24. Yu Y, Su L, Wang X, Wang X, Xu C. Association between Helicobacter pylori infection and pathological changes in the gastric mucosa in Chinese children. Intern Med 2014; 53: 83-88.

25. Flagstadt G, Helgeland H, Markestad T. Faecal calprotectin concentration in children with functional gastrointestinal disorders diagnosed according to pediatric Rome III criteria. Acta Pediatr 2010; 99: 734-737.

26. Lahat A, Kopylov U, Neuman S, et al; on behalf of the Israeli IBD research Network (IIRN) Helicobacter pylori prevalence and clinical significance in patients with quiescent Crohn's disease. BMC Gastroenterol 2017; 17: 27.

27. Summerton CB, Longlands MG, Wiener K, Shreeve DR. Faecal calprotectin: a marker of inflammation throughout the intestinal tract. Eur J Gastroenterol Hepatol 2002; 14: 841-845. 